\title{
Delayed Post-hypoxic Leukoencephalopathy with Neuroradiological Recovery
}

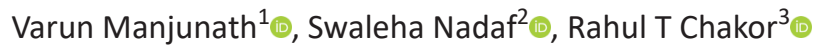 \\ Keywords: Arylsulfatase, Delayed post-hypoxic encephalopathy, Demyelination, Hypoxia, Metabolic, Toxic. \\ Indian Journal of Critical Care Medicine (2021): 10.5005/jp-journals-10071-24014
}

Sir,

Delayed post-hypoxic leukoencephalopathy (DPHL) is a delayed manifestation of hypoxia. Acute severe hypoxia typically affects gray matter, and mild to moderate prolonged hypoxia leads to DPHL. ${ }^{1}$ Delayed, extensive white matter abnormalities due to hypoxia may often create diagnostic and prognostic dilemma when patient presents with neurocognitive syndrome in the second phase. DPHL can have spontaneous clinical and neuroradiological recovery. ${ }^{2}$ DPHL with biphasic presentation and extensive leukoencephalopathy with near-complete clinicoradiological recovery make our case noteworthy. The majority of DPHL cases reported are associated with carbon monoxide intoxication; the incidence is approximately $2.75 \% .^{1,3}$

A 76-year-old male presented with acute pneumonia in respiratory distress with $\mathrm{SpO}_{2}-84 \%$ on bag and mask support $\mathrm{FiO}_{2}$ 95\%. He was encephalopathic on presentation (Glasgow Coma Scale 7/15) and was later kept on mechanical ventilator with midazolam sedation. Over the next 2 days, midazolam infusion was tapered and stopped. He gradually improved over the next 10 days. He became independent for activities of daily living (ADL). Few weeks later, he developed progressive cognitive decline and bowel/bladder incontinence. He was not able to carry out any of the day-to-day activities. On examination, his vital parameters were normal. He was inattentive, apathic, and disoriented. MiniMental State Examination (MMSE) was 11/30, with points lost in attention and registration. Frontal assessment battery (FAB) score was $3 / 18$. He had symmetrical extrapyramidal signs with slow, short shuffling gait.

Hemogram, sugar, liver and renal function test, arterial ammonia, TSH, anti-TPO, vitamin B12, HIV, and VDRL were normal. Toxicology screening was negative. Electroencephalography showed mild diffuse encephalopathy. Cerebrospinal fluid (CSF) routine and meningoencephalitis panel were negative. Lactate and pyruvate levels were normal. Serum neuromyelitis optica (NMO), myelin oligodendrocyte glycoprotein (MOG) antibodies, antinuclear antibody (ANA), antineutrophil cytoplasmic antibody (ANCA), angiotensin-converting enzyme (ACE), autoimmune and paraneoplastic encephalitis workup were negative.

Magnetic resonance imaging (MRI) brain showed T2, fluidattenuated inversion recovery (FLAIR) symmetrical diffuse hyperintense signals predominantly in central white matter of bilateral frontal, temporal, parieto-occipital lobes. There was sparing of internal capsule, periventricular region, cerebellum, and brainstem. There was symmetrical diffusion restriction with ADC drop and no contrast enhancement.

\footnotetext{
${ }^{1-3}$ Department of Neurology, Topiwala National Medical College, Mumbai, Maharashtra, India
}

Corresponding Author: Varun Manjunath, Department of Neurology, Topiwala National Medical College, Mumbai, Maharashtra, India, Phone: +91 9611189964, e-mail: varunmanjunath13@gmail.com

How to cite this article: Manjunath V, Nadaf S, Chakor RT. Delayed Post-hypoxic Leukoencephalopathy with Neuroradiological Recovery. Indian J Crit Care Med 2021;25(11):1326-1328.

Source of support: Nil

Conflict of interest: None

Differential diagnoses considered were as follows:

- Toxic leukoencephalopathy ${ }^{4}$ (carbon monoxide poisoning, inhaled heroin, methanol, methotrexate toxicity).

- MRI in carbon monoxide poisoning shows confluent bilateral relatively symmetric white matter hyperintensity on $\mathrm{T} 2$ and FLAIR sequences with associated restricted diffusion; ${ }^{4}$ however, our patient's carboxyhemoglobin levels were normal.

- Inhalational encephalopathy due to heroin (chasing the dragon sign) affects cerebellum, brainstem, parieto-occipital white matter, and posterior limb of internal capsule. ${ }^{4}$ Our patient had no such history of toxin or drug exposure, and toxicology screening was negative.

- Metabolic leukoencephalopathy ${ }^{5}$ [extrapontine myelinolysis (EPM), septic encephalopathy]. However, EPM or septic encephalopathy has more predilection for basal ganglia and thalamus and deep/periventricular white matter. ${ }^{6}$ Our patient had normal electrolytes, liver and renal function tests. There was no evidence of sepsis during the phase of delayed neurocognitive decline.

- Inflammatory demyelinating diseases such as symmetrical and extensive white matter involvement with diffusion restriction without contrast enhancement were considered atypical for multiple sclerosis (MS) or acute disseminated encephalomyelitis (ADEM). CSF study was normal including oligoclonal bands.

- Posterior Reversible Encephalopathy Syndrome (PRES)-The involvement of $U$ fibers and cortex is typical for PRES. ${ }^{2} \mathrm{MRI}$ in our patient was odd for PRES. There was no hypertension, and there were no etiological factors favoring PRES in our patient.

- Mitochondrial encephalopathy-CSF lactate-pyruvate negative.

() The Author(s). 2021 Open Access This article is distributed under the terms of the Creative Commons Attribution 4.0 International License (https://creativecommons. org/licenses/by-nc/4.0/), which permits unrestricted use, distribution, and non-commercial reproduction in any medium, provided you give appropriate credit to the original author(s) and the source, provide a link to the Creative Commons license, and indicate if changes were made. The Creative Commons Public Domain Dedication waiver (http://creativecommons.org/publicdomain/zero/1.0/) applies to the data made available in this article, unless otherwise stated. 

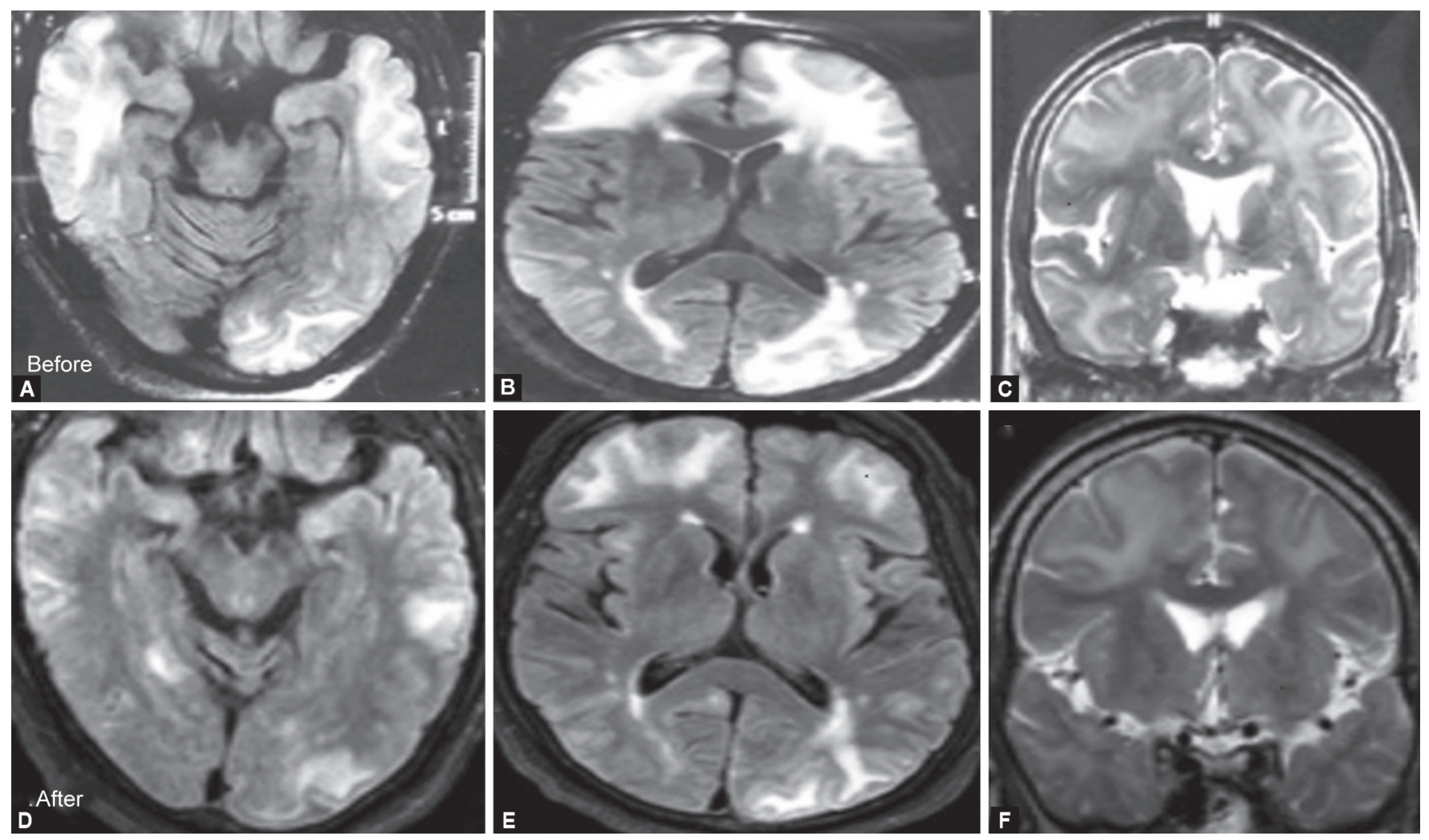

Figs 1 A to F: Before (A)_FLAIR axial $(1,2)$ and T2 coronal (3) images show symmetrical hyperintense signals in subcortical white matter of temporal, occipital lobes and bilateral frontal lobes with slightly asymmetric hyperintensities (left > right) in bilateral parieto-occipital regions. After 3 months; (B) - FLAIR axial $(4,5)$ and T2 coronal (6) images show significant resolution of hyperintensities in white matter of temporal, occipital, and frontal regions

DPHL was thought of as the possibilities of sepsis, PRES, ADEM, toxic, mitochondrial encephalopathy were ruled out. He was given supportive therapy. He showed spontaneous improvement over the next few weeks and became independent for ADL. His MMSE was $28 / 30$ and FAB $14 / 18$ over the next 3 months. He had mild extrapyramidal signs. Follow-up MRI after 4 months showed significant resolution of white matter hyperintensities (Fig. 1).

Multiple hypotheses have been proposed for DPHL. Cell death after cerebral ischemia is considered to be necrotic in nature, but recent studies have revealed that apoptosis, a delayed form of cell death, may be the reason of cell death in white matter ${ }^{1}$. DPHL is characterized by selective demyelination due to apoptosis of oligodendrocytes in arterial border zones.

DPHL has also been reported in the setting of deficiency of arylsulfatase $A$. A relative reduction of arylsulfatase $A$ activity may occur in $15 \%$ of general population (pseudodeficiency) due to allelic mutations of the gene that gives rise to metachromatic leukodystrophy. ${ }^{5}$ Asymptomatic pseudodeficiency might predispose a small percentage of patients to delayed white matter loss after hypoxic event.

During the acute prolonged low to moderate grade hypoxic event, patients are encephalopathic due to global hypoperfusion. As the oxygenation improves, there is recovery of sensorium. But, due to initial low-grade hypoxic insult, there may be delayed apoptosis and demyelination in some patients leading to DPHL. Imaging may be normal during initial hypoxic event as well as during interval phase.
Delayed and purely white matter involvement after hypoxicischemic insult may imply that degree of hypoxia was not profound..$^{5}$ Hence, prognosis may be better than acute, severe hypoxic encephalopathy affecting gray matter (cortex, basal ganglia). Supportive care and antioxidants are the mainstay of treatment. ${ }^{7}$ Prognosis of DPHL is usually variable. Occasionally full or partial recovery is noticed.

To conclude, in the current COVID pandemic situation, there are increased cases of DPHL in patients requiring prolonged oxygen support. ${ }^{7}$ In the absence of awareness of this entity, patients are being worked up extensively and given a poor prognosis. DPHL is a rare, under-recognized form of post-hypoxic leukoencephalopathy with relatively better prognosis. Due to the late clinical and imaging manifestations, it might be overlooked by the unaware clinician.

\section{Author Contributions}

Dr Swaleha Nadaf was involved in study conception and design; analysis and interpretation and acquisition of data. Dr Rahul T Chakor was involved in critical revision of the manuscript for important intellectual content, discussion, and final approval. Dr Varun Manjunath was involved in the review of literature and discussion.

\section{OrCID}

Varun Manjunath @ https://orcid.org/0000-0001-6882-7846

Swaleha Nadaf (1) https://orcid.org/0000-0002-8953-9875

Rahul T Chakor (ํ) https://orcid.org/0000-0003-4907-9154 


\section{References}

1. Katyal N, Narula N, George P, Nattanamai P, Newey CR, Beary JM. Delayed post-hypoxic leukoencephalopathy: a case series and review of the literature. Cureus 2018;10(4):e2481. DOI: 10.7759/cureus.2481. Available from: https://www.cureus.com/articles/11882-delayedpost-hypoxic-leukoencephalopathy-a-case-series-and-review-ofthe-literature.

2. Beeskow $A B$, Oberstadt $M$, Saur D, Hoffmann K-T, Lobsien D. Delayed post-hypoxic leukoencephalopathy (dphl) —an uncommon variant of hypoxic brain damage in adults. Front Neurol 2018;9:708. DOI: 10.3389/fneur.2018.00708. Available from: https://www.frontiersin. org/article/10.3389/fneur.2018.00708/full.

3. Choi S. Delayed neurologic sequelae in carbon monoxide intoxication. Arch Neurol 1983;40(7):433-435. DOI: 10.1001/ archneur.1983.04050070063016.
4. Geraldo AF, Silva C, Neutel D, Neto L, Albuquerque L. Delayed leukoencephalopathy after acute carbon monoxide intoxication. J Radiol Case Rep 2014;8(5):1-8. DOI: 10.3941/jrcr.v8i5.1721.

5. Van der knaap MS, Valik J. magnetic resonance of myelin, myelination and myelin disorders: delayed post hypoxic leukoencephalopathy 2nd edition. 29th June 2013. p. 387-391.

6. Mazón M, Montoya-Filardi A. Report of a case of delayed posthypoxic leukoencephalopathy: a peculiar image. Neurología (Engl Ed) 2020;35(3):213-216. DOI: 10.1016/j.nrl.2018.01.002.

7. King F, Morris NA, Schmahmann JD. Delayed posthypoxic leukoencephalopathy: improvement with antioxidant therapy. Case Rep Neurol 2015;7(3):242-246. DOI: 10.1159/000441892. 